\title{
Otolaryngological features in children with trisomy 21: a study of 171 patients at the Hospital Infantil de México Federico Gómez
}

\author{
Natalia Peraza, Carlos De La Torre, Alicia Álvarez and Perla Villamor* \\ Servicio de Otorrinolaringología Pediátrica, Hospital Infantil de México Federico Gómez, Mexico City, Mexico
}

\begin{abstract}
Background: Children with trisomy 21 face a wide range of conditions in the head and neck region, for which it is important that physicians are aware and have a strong understanding of the ear, nose, and throat (ENT) disorders, and their management as well. Methods: Retrospective case series of pediatric patients with trisomy 21. The spectrum of otolaryngological manifestations, their management, and outcomes of each case were analysed. Results: One hundred and seventeen pediatric patients were included. The mean age was $7.2 \pm 4.2$ years. More than half of the patients (63\%) had hearing loss $(H \mathrm{H})$. The most frequent presentation was conductive $H L$, predominating the mild and bilateral type. The most common otological manifestations found were external ear canal stenosis and Eustachian tube dysfunction. Up to $75 \%$ of the patients with otologic involvement required some surgical procedure. The most common rhinological manifestations were chronic rhinosinusitis and allergic rhinitis. Obstructive sleep apnea (OSA) was present in 30\% of all patients, which main treatment was-tonsillectomy, followed by continuous positive and biphasic positive airway pressure treatments. Less than $5 \%$ of the patients presented a laryngeal compromise. Conclusions: Pediatric patients with trisomy 21 systematically should be referred to periodic ENT assessment due to the high incidence of manifestations in this region. Timely treatments should be offered in order to improve the health and the quality of life of the patient.
\end{abstract}

Key words: Trisomy 21. Down syndrome. Children. Otolaryngology.

\section{Características otorrinolaringológicas en niños con trisomía 21: un estudio de 171 pacientes en el Hospital Infantil de México Federico Gómez}

\section{Resumen}

Introducción: Los niños con trisomía 21 enfrentan una amplia gama de problemas en la región de la cabeza y el cựello, por lo cual es importante reconocer las manifestaciones otorrinolaringológicas que presentan, así como su apropiado manejo. Métodos: Estudio de serie de casos retrospectivo de pacientes pediátricos con trisomía 21. De cada caso se anălizó el espectro de manifestaciones otorrinolaringológicas, el manejo establecido y los resultados. Resultados: Se incluyeron 171 niños. La edad media de la primera valoración por otorrinolaringología en la institución fue de $7.2 \pm 4.2$ años. Las mañifestaciones otológicas más frecuentes fueron la estenosis del conducto auditivo externo y la disfunción de la trompa de Eustaquio. Más de la mitad de los pacientes (63\%) presentaron hipoacusia, principalmente de tipo conductivo bilateral, y

Correspondence:

*Perla Villamor

E-mail: perla.villamor@gmail.com

2444-3409/O 2018. Hospital Infantil de México Federico Gd
Date of reception: 23-09-2018

Date of acceptance: 14-01-2019

DOI: 10.24875/BMHIME.M19000059
Available online: 14-01-2020 Bol Med Hosp Infant Mex. 2019;76:81-88 www.bmhim.com 
el $75 \%$ de los pacientes con afectación otológica requirieron algún procedimiento quirúrgico. Las manifestaciones rinológicas más comunes fueron la rinosinusitis crónica y la rinitis alérgica. La apnea obstructiva del sueño estuvo presente en el30\% de los pacientes. El tratamiento principal fue la amigdalectomía, seguida del tratamiento con dispositivos de presión pósitiva de la vía aérea. Menos del 5\% de los pacientes presentaron un compromiso laríngeo. Conclusiones: Los pacientes pediátricos con trisomía 21 deben ser remitidos sistemáticamente a una evaluación otorrinolaringológica periódica, débido a la alta incidencia de manifestaciones en esta región. Se deben ofrecer tratamientos oportunos para mejorar su saftud y calidad de vida.

Palabras clave: Trisomía 21. Síndrome de Down. Niños. Otorrinolaringología.

\section{Introduction}

Trisomy 21 is one of the most common genetic disorders and the most critical cause of intellectual disability. ${ }^{1}$ It was first described in 1866 by John L. Down. It is most commonly caused by a nondisjunction error, which results in three copies of chromosome $21 .^{2}$ The incidence of trisomy 21 is affected by the maternal age and varies in the population (between 1 in 319 and 1 in 1,000 live births). ${ }^{3}$ It has high phenotypic variability and genetic complexity. ${ }^{4}$ The recent improvement in medical care has increased the life expectancy of patients with Down syndrome, for which an average life expectancy of 55 years is reported in developed countries. ${ }^{5}$

Several features of trisomy 21 are expressed in the head and neck region. These can cause sequelae that prevent patients from reaching their maximum developmental capacities, as well as hamper their quality of life. ${ }^{6}$ As medical science advances and these patients achieve long lifespans, physicians must have the awareness and a solid understanding of the otorhinolaryngological manifestations of children with Down syndrome, as well as its management.

This study aimed to present a series of cases of pediatric patients with trisomy 21 , their spectrum of otorhinolaryngological manifestations, clinical and sociodemographic characteristics, management, and results.

\section{Methods}

A retrospective case series study was conducted.

\section{Data collection}

A database was created with the medical records of pediatric patients $(<18$ years) with trisomy 21 confirmed by karyotype, assessed at the ENT Otorhinolaryngology Service of the Hospital Infantil de México Federico Gómez during the period from January 2007 to January 2017.
The following data were recorded for each patient: demographic characteristics, associated systemic diseases, primary and secondary otorhinolaryngological diagnoses, surgical management, postoperative outcomes, and complications. The most comprehensive and early audiogram was used to characterize the hearing ability and, in cases of hearing loss, to determine its type, severity, and laterality. Within the institutional protocol, patients younger than two years old are evaluated by brainstem auditory-evoked potentials (BAEP) for bone-conducted and air-conducted stimuli. At an older age, patients are evaluated by tonal audiometry by both air and bone-conducted stimuli through play or behavioral observation. For the patients otder than two years in whom it is not possible to perform the audiometry, the evaluation continues with BAEP.

\section{Data management and statistical analysis}

The data was collected in forms and analyzed using the Stata statistical software, version 13 (College Station, TX, StataCorp LP). Continuous variables are-depicted as mean \pm standard deviation, and categorical variables are presented as counts.

\section{Ethics}

This study was carried out following the World Medical Association Declaration of Helsinki on ethical principles for medical research.

\section{Results}

One hundred and seventy-one pediatric patients with trisomy 21 confirmed by genetic evaluation and karyotype were included. Among the study population, the average age at the moment of the first assessment by the otorhinolaryngology service was $7.2 \pm 4.2$ years (range: 1 month to 18 years), and $42.7 \%$ of the cases were females, with a 1:1.4 ratio (Table 1). Associâted morbidities included overweight/obesity, cardiovascular 
Table 1. Characteristics of the patients

\begin{tabular}{|l|c|}
\hline Characteristics & $\mathbf{N}=\mathbf{1 7 1} \mathbf{n}(\%)$ \\
\hline $\begin{array}{l}\text { Age at first assessment by the } \\
\text { otolaryngology service of the HIMFG } \\
\text { (years } \pm \text { SD) }\end{array}$ & $7.1 \pm 4.2$ \\
\hline $\begin{array}{l}\text { Gender } \\
\text { Male }\end{array}$ & \\
\hline Female & $98(57.3)$ \\
\hline $\begin{array}{l}\text { Associated morbidity } \\
\text { Overweight/obesity }\end{array}$ & $73(42.7)$ \\
\hline Cardiopathy & $139(81.3)$ \\
Hypothyroidism & $124(72.5)$ \\
Diabetes & $48(28.1)$ \\
Cleft lip and cleft palate & $4(2.4)$ \\
Acute lymphoblastic leukemia & $4(2.4)$ \\
\hline
\end{tabular}

HIMFG, Hospital Infantil de México Federico Gómez; SD, standard deviation.

disease, and hypothyroidism, followed by diabetes, cleft lip and palate, and acute lymphoblastic leukemia (Table 1).

Regarding the audiological findings, $63 \%$ of patients presented hearing loss. The most frequent presentation was a conductive hearing loss (92\% of the cases), predominantly of the mild and bilateral type. Furthermore, sensorineural hearing loss accounted for only $18 \%$ of cases, although it had a more severe presentation. Profound bilateral hearing loss was the most common type of sensorineural hearing loss. Of the total of patients, $15.05 \%$ (14 patients) were rehabilitated with hearing aids. However, it was not possible to establish in which cases the aid was given unilaterally or bilaterally. None of the patients was rehabilitated using CROS (contralateral routing of signals) or BiCROS systems, bone conduction hearing devices, or cochlear implants (Table 2).

The central pathology of the external ear was the narrowing of the external auditory meatus (EAM), which occurred in $98.24 \%$ of the patients, with a spectrum that ranged from partial narrowing to stenosis. Furthermore, the EAM narrowness was the only otologic condition in $46 \%$ of patients. The most common otologic manifestations found after EAM stenosis were eustachian tube dysfunction without effusion and otitis media with effusion, followed by chronic otitis media without cholesteatoma and chronic otitis media with cholesteatoma (Table 2). Up to $75 \%$ of patients with otologic involvement required some surgical procedure, while the remaining $25 \%$ were managed only with medical treatment (eustachian tube exercises, nasal spray, systemic antibiotic therapy, and quinolone ear drops). The principal surgery performed to treat otologic involvement
Table 2. Manifestations, treatments, and procedures for the management of otological diseases

\begin{tabular}{|c|c|}
\hline Characteristic & $N=171 n(\%)$ \\
\hline $\begin{array}{l}\text { External ear pathology } \\
\text { Narrow external auditory canal } \\
\text { Microtia }\end{array}$ & $\begin{array}{l}168(98.25) \\
18(10.52)\end{array}$ \\
\hline $\begin{array}{l}\text { Middle ear pathology } \\
\text { Eustachian tube dysfunction without effusion } \\
\text { Otitis media with effusion } \\
\text { Chronic otitis media without cholesteatoma } \\
\text { Chronic otitis media with cholesteatoma }\end{array}$ & $\begin{array}{l}39(22.81) \\
37(21.64) \\
11\left(6.43 \frac{5}{5}\right. \\
6\left(3.51 \frac{\underline{6}}{5}\right.\end{array}$ \\
\hline $\begin{array}{l}\text { Audiological findings } \\
\text { Normal hearing } \\
\text { Total hearing loss of any type } \\
\text { Conductive hearing loss } \\
\text { Mild } \\
\text { Moderate } \\
\text { Severe } \\
\text { Profound } \\
\text { Bilateral } \\
\text { Unilateral }\end{array}$ & 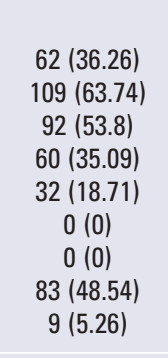 \\
\hline $\begin{array}{l}\text { Sensorineural hearing loss } \\
\text { Mild } \\
\text { Moderate } \\
\text { Severe } \\
\text { Profound } \\
\text { Bilateral } \\
\text { Unilateral }\end{array}$ & $\begin{array}{l}18(10.52) \\
2(1.17) \\
0(0) \lessgtr \\
3(1.75) \\
12(7.02) \\
12(7.02) \\
5(2.92)\end{array}$ \\
\hline $\begin{array}{l}\text { Type of treatment* } \\
\text { Medical management } \\
\text { Adaptation to hearing aids } \\
\text { Myringotomy with ventilation tube placement } \\
\text { Myringotomy without ventilation tube } \\
\text { placement }\end{array}$ & 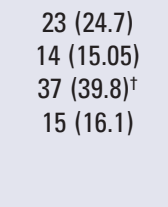 \\
\hline $\begin{array}{l}\text { Canal wall up mastoidectomy } \\
\text { Tympanoplasty } \\
\text { Adenoidectomy (to treat otological problems) }\end{array}$ & $\begin{array}{l}7(7.5) \frac{\circ}{\circ} \\
6(6.5) \cup \\
5(5.4) !\end{array}$ \\
\hline Age at the time of surgery (years \pm SD) & $4.6 \pm 3 . \underline{\tilde{z}}$ \\
\hline $\begin{array}{l}\text { D, standard deviation. } \\
\text { Percentage based on the total number of patients with otolo } \\
N=93 \text { ). } \\
\text { A total of } 47 \text { myringotomies with ventilation tube placement } v \\
\text { atients. The average number of ventilation tubes per patient } \\
\text { I 1.145-1.455). }\end{array}$ & 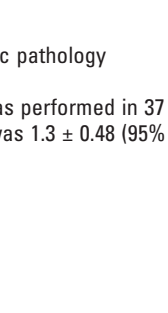 \\
\hline
\end{tabular}
was myringotomy, with or without ventilation tube placement, which was performed in $56 \%$ of patients with otologic involvement. An average of $1.27 \pm 0.48$ myringotomies was performed per patient. The average-age of ventilation tube placement was $3.81 \pm 2.87$ years (range of 1 to 14 years). Of the 52 patients who underwent myringotomy, six had otorrhea during follow-up, and two had a residual tympanic perforation. Tympanoplasty, mastoidectomy, and adenoidectomy (specific for the management of otological problems) were performed in less than $10 \%$ of the patients (Table 2). 
The most common nasosinusal manifestations were chronic rhinosinusitis and allergic rhinitis. Two cases of invasive fungal rhinosinusitis associated with acute lymphoblastic leukemia were also documented. The main treatments for the nasosinusal manifestations were nasal washes, nasal sprays, antihistamines, and systemic antibiotics, as well as adenoidectomy (specific for the treatment of chronic rhinosinusitis). Functional endoscopic surgery (FES) and turbinoplasty were performed in less than $10 \%$ of cases with nasosinusal involvement (Table 3).

Macroglossia was the principal manifestation in the oral cavity and the oropharynx, with a prevalence of $69 \%$. Respiratory sleep disorders were present in 30\% of patients. Obstructive tonsillar hypertrophy was evident in $24 \%$ of the cases. The primary treatment for obstructive sleep disorders was tonsillectomy, followed by medical treatment, which consisted mainly of using positive airway pressure (CPAP) and bilevel positive airway pressure (BiPAP) devices. $11 \%$ of patients who underwent tonsillectomy persisted with nocturnal obstructive symptoms despite surgical treatment; these symptoms were defined as respiratory pauses witnessed by the caregiver or a health professional, accompanied by snoring. Moreover, delayed post-tonsillectomy hemorrhage occurred in one patient, who was successfully managed with hemostasis in the operating room (Table 4).

Less than $5 \%$ of patients presented a laryngeal compromise. Four patients had laryngomalacia, of which three were satisfactorily treated with medical treatment and follow-up care. Only one patient required supraglottoplasty. Two patients had subglottic stenosis that required tracheostomy and endoscopic laryngeal dilatations to secure the airway. During follow-up, one of the two patients was successfully decannulated (Table 5).

\section{Discussion}

Considering that neural plasticity, language development, and the beginning of schooling occur before seven years of age, the initial assessment by the otorhinolaryngology service was at a late age in this series of cases. For this reason, timely referral protocols to an ENT-specialist should be established for patients with diagnosis or suspicion of trisomy 21. The recommendations for diagnosis and rehabilitation of hearing loss endorsed by the Joint Committee on Infant Hearing indicate that the diagnosis should be made before three months of age, and the rehabilitation should start
Table 3. Nasosinusal manifestations

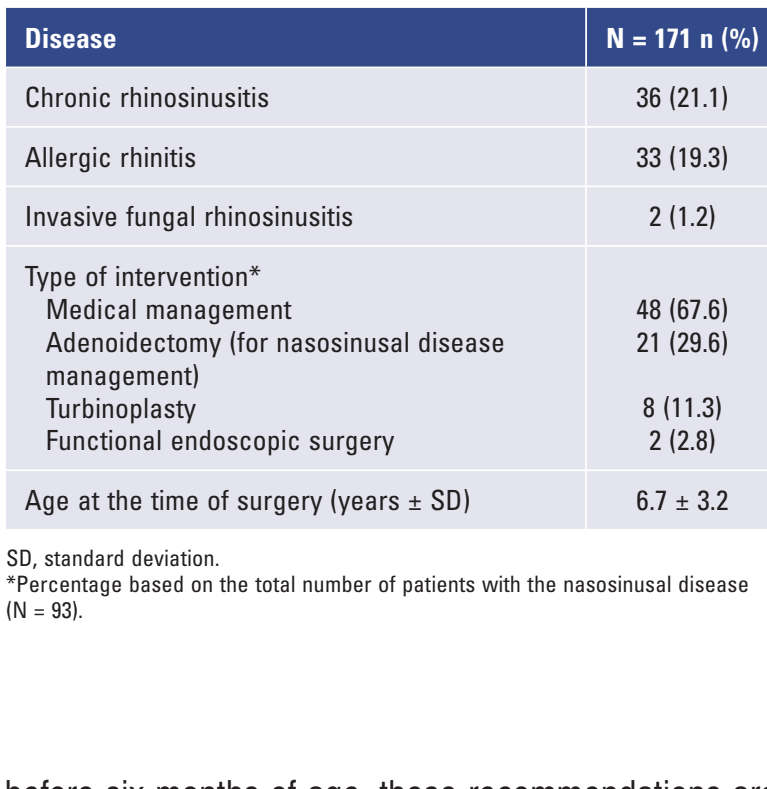

before six months of age, these recommendationsiare even more relevant in trisomy 21 population as they have a higher hearing loss prevalence.

Children with trisomy 21 experience a higher prèvalence of comorbidities compared to individuals without the syndrome. Kong et al. reported that upper respiratory tract infections, otitis media, nutritional and metabolic symptoms, and global developmental delay were among the most common comorbidities in Down syndrome. ${ }^{7}$ In this series, overweight/obesity, cardiovascular disease, and hypothyroidism were the most prevalent comorbidities.

Regarding otorhinolaryngological manifestations, in their study of 50 patients with Down syndrome, Lau et al. found that a third of the cases had some hearing loss, and of these, $72 \%$ were of the conductive type. ${ }^{8}$ Kreicher et al., using the AudGenDB audiological and genetic database of 1,088 patients with trisomy 21 , found that $67 \%$ of the patients had a hearing deficit that persisted in all available audiograms. ${ }^{9}$ In the present study, $63.7 \%$ of the patients had hearing loss, which was mainly conductive, bilateral, and mild. However, in cases of sensorineural hearing loss, the compromise was predominantly profound and bilateral. The Angerican Academy of Pediatrics recommends that a child with trisomy 21 who has passed his or her neonatal screening test should undergo electrophysiological hearing tests every six months until pure-tone audiometry can be performed (usually after four years old) iand after that, annual follow-up audiometry is advised. ${ }^{10} \mathrm{dt}$ is possible that the low incidence of sensorineural hearing loss in the present series, as well as the apparenfabsence of mixed hearing loss, are due to the detection 
Table 4. Oral cavity and pharyngeal manifestations

Disease

Macroglossia

Tonsillar hypertrophy

Symptoms of respiratory sleep disorder without diagnosis with PSG

OSA confirmed by PSG

Type of intervention*

Medical management including positive airway pressure devices (CPAP/ BiPAP)

Tonsillectomy

Complications ${ }^{\dagger}$

Obstructive symptoms after the tonsillectomy

Bleeding after tonsillectomy

Age at the time of surgery (years \pm SD)
$\mathbf{N}=171$ n (\%)

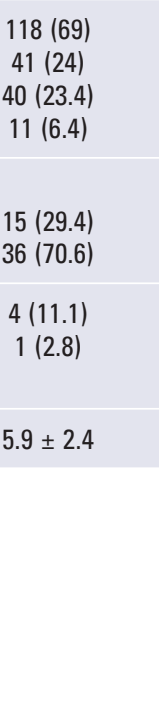

Table 5. Laryngeal manifestations

\section{Disease}

Laryngomalacia

Subglottic stenosis

Type of intervention*

Medical management

Tracheostomy

Endoscopic management with laryngeal

dilation

Supraglottoplasty

Age at the time of surgery (years \pm SD)

SD, standard deviation

*Percentage based on the total number of patients with laryngeal diseases $(N=6)$. †Only patients with laryngomalacia.

method used in patients older than two years (play audiometry or behavioral observation). These techniques could underestimate, in some cases, a mild sensorineural component or a unilateral hearing loss with a contralateral normoacoustic ear. It should be taken into account that auditory evaluation is a challenge in this population, since children with Down syndrome may have little attention during behavioral tests and an inconsistent voluntary response, which makes it difficult to differentiate the type and side of hearing loss. Therefore, electrophysiological tests, such as brainstem auditory evoked potentials, are particularly useful in this context, since they can assess auditory performance, regardless of the age and mental state of the patient. ${ }^{8}$

In this series, $15.05 \%$ of patients were rehabilitated with adaptation to hearing aids, although it was not possible to establish the cases in which the adaptation was performed unilaterally or bilaterally. None of the patients was rehabilitated by CROS or BiCROS systems, bone conduction hearing devices, or cochlear implants. The use of standard hearing aids is complicated in these patients since many of these children have learning problems and do not cooperate with a hearing device. Also, conventional aids can exacerbate external otitis and coexisting otitis media. ${ }^{11} \mathrm{~A}$ recent study showed that children with Down syndrome had a significant improvement in their daily activities, learning, and concentration with bone conduction hearing devices such as $B A H A^{\circledR}$. The BAHA ${ }^{\circledR}$ device alsooimproved the development of auditory skills and reduced the distraction time in these patients, which is whyit is considered a useful device in auditory rehabilitation after conventional hearing aids have failed. ${ }^{11}$

Most children with trisomy 21 have otologic conditions, which include narrowing of the auditory meatus, chronic otitis media, and hearing loss, all of them require evaluation and treatment by an otolaryngologist. ${ }^{12}$ In the present study, $46 \%$ of patients had EAM stennosis, and $54 \%$ had another type of additional ototogic compromise. EAM stenosis has been described if 40 to $50 \%$ of patients with Down syndrome; this condition facilitates cerumen, hair, and foreign body impaction, which is difficult to eliminate, contributing to conductive hearing loss. ${ }^{13,14}$ Although a universally accepted definition of stenosis has not been established, a study of patients with congenital aural stenosis defined it as a canal diameter $\leq 4 \mathrm{~mm}^{15}$ In a recent study of $130 \mathrm{opa}$ tients with trisomy 21, Dy and Lapeña determined that the prevalence of auditory canal stenosis in their study population was $72.3 \%{ }^{16}$ 
Facial dysmorphia leads to abnormalities of the ear and upper respiratory tract, including EAM stenosis and eustachian tube dysfunction. ${ }^{17}$ This phenotype, combined with an inadequate immune function, results in the development of upper respiratory tract infections. ${ }^{18}$ Moreover, generalized hypotonia can lead to the dysfunction of the tensor veli palatini muscle, which increases the risk of acute otitis media and chronic effusion due to eustachian tube collapse. ${ }^{19}$ When eustachian tube dysfunction remains undiagnosed, late sequelae may appear, such as tympanic perforation, retraction pockets, and cholesteatoma. It has been described that $7.1 \%$ of patients with trisomy 21 develop cholesteatoma. ${ }^{20,21}$ In this series, only $3 \%$ of patients had chronic otitis media with cholesteatoma, and all were treated successfully with a canal wall up mastoidectomy. It should be noted that no canal wall down mastoidectomy was reported in the studied patients. The delay in the diagnosis and treatment of a cholesteatoma could cause a labyrinthine fistula, labyrinthitis, facial nerve paralysis, and intracranial complications. ${ }^{20,21}$

In a case series of 676 children with eustachian tube dysfunction, including 46 with trisomy 21, Crowson et al. reported that intranasal fluticasone delayed the time needed for placement of ventilation tubes; however, this finding did not apply for children with Down syndrome..$^{22}$ In the present series, the main surgery performed to treat otological compromise was myringotomy with or without ventilation tube placement $(56 \%$ of cases). This surgery is the most common pediatric surgical procedure worldwide. However, ventilation tube placement may be difficult or even impossible in children with trisomy 21 , since the morphological features of the syndrome include rather narrow ear canals. It is possible that early placement of ventilation tubes in children with otitis media with effusion, may lead to better results by diminishing scars and permanent hearing damage; however, a prospective study is needed to evaluate this possibility. Furthermore, it has been shown that the surgical placement of ventilation tubes improves the quality of life significantly. In this study, the average number of myringotomies per patient was $1.27 \pm 0.48$. One of the risks of repeated placement of ventilation tubes is the incidence of $17 \%$ of tympanic membrane perforation. ${ }^{23}$ These patients also have the risk of permanent tympanic perforation after the tube extrusion, which is due to a thin tympanic membrane that lacks its lamina propria, a layer rich in blood vessels and collagen fibers. ${ }^{13}$ In the present series, six of the 52 patients who underwent myringotomy presented otorrhea during follow-up, and two had a residual tympanic perforation, which was managed successfully with tympanoplasty. Ghadersohi et al. recently demonstrated a global tympanoplasty success rate of $70.9 \%$ (closure rate of $54.8 \%$ ). Based on this analysis, the type of tube, specifically a long-acting tube (T-tube), wasnot associated with persistent perforation. The authors supported cartilage tympanoplasty in this population because it better resists the negative pressure of the middle ear generated by the Eustachian tube. ${ }^{23}$ Amplification devices could be used to improve hearing but conventional BTE hearing aids are often not tolerated. Moreover, it has been shown that adenoidectomy increases the benefit of the insertion of ventilation tựbes in certain groups of children without trisomy $21.24 \stackrel{\circ}{\circ}$

Patients with trisomy 21 may suffer from recurfent sinusitis due to abnormal development of the frontal, maxillary, and sphenoid sinuses. Hypoplasia and non-complete pneumatization are common in this population. On the other hand, delayed maturation of the immune system or ciliary dyskinesia can also cause chronic sinusitis. ${ }^{14}$ In this study, chronic rhinosinusititis and allergic rhinitis were detected as the most common rhinological manifestations, with two cases of invasive fungal rhinosinusitis associated with acute lymphobtastic leukemia. In patients with Down syndrome, rhinorrhea may improve with age, due to the enlargement of the anatomical abnormalities. The treatment should be similar to other cases and includes antibiotics, nasal steroids, decongestants, and antihistamines. If sinusijtis persists, surgical alternatives may include adenoidectomy and functional endoscopic sinus (FES) surgery. ${ }^{13}$ In this study, the vast majority of patients were successfully treated only with medical treatment, and in only $2.8 \%$ of cases, the FES was performed.

Macroglossia was the primary manifestation of the oral cavity and the oropharynx, with a $69 \%$ prevalence. Respiratory sleep disorders were present in $30 \%$ of patients. Obstructive sleep apnea (OSA) is a common comorbidity in this population, with an estimated prevalence between 30 and $70 \% .{ }^{25}$ In a recent meta-analysis, Lee et al. found that the OSA prevalence based on an apnea-hypopnea index $(\mathrm{AHI})>1,1.5,2,5$ and 10 events/h were $69 \%, 76 \%, 75 \%, 50 \%$, and $34 \%$, respectively, in children with trisomy 21 who underwent polysomnography. In this study, the subgroup analyses revealed no statistically significant differences. ${ }^{26}$

Craniofacial features, including maxillary hypoplásia, macroglossia, and oropharyngeal hypotonia, contribute to the respiratory alterations during sleep. Children with Down syndrome have a high risk of OSA, not onlybecause of their morphological differences but calso 
because of their tendency toward obesity. Furthermore, the multiple endocrine abnormalities presented in this syndrome, such as hypothyroidism and type 1 diabetes mellitus, are associated with weight gain and obesity. ${ }^{27}$ In the present series, only $6.4 \%$ of the patients underwent diagnostic polysomnography (PSG). The low rate of PSG studies could be due to their high cost, which may generate an OSA underdiagnosis in this series. Laboratory-based PSG is the gold standard for the diagnosis of pediatric OSA, although the cost and availability often limit its use. Given the difficulty of performing a PSG in all patients due to its high costs and low accessibility, it would be advisable to consider alternative studies, although they are not considered as a gold standard. Examples of these are respiratory polygraphy, and drug-induced sleep endoscopy, which have a much lower cost to timely assess the obstruction of these patients before performing any surgery. Also, they determine the level of obstruction of the upper airway. The above is relevant if one considers that the obstruction can be multilevel or may be different from the one suspected only by the physical examination. The realization of this study is possible during anesthetic induction with flexible laryngoscopy before a scheduled surgical procedure. ${ }^{28}$

Farhood et al. showed a statistically significant improvement in PSG values in patients with trisomy 21 and OSA who underwent a tonsillectomy in a systematic review. However, the majority of patients could not achieve an $\mathrm{AHI}<1 .{ }^{29}$ In the present study, the persistence rate of obstructive symptoms after tonsillectomy was $11 \%$. However, because the patients did not have pre-surgical and post-surgical routine polysomnography, the actual percentage of post-tonsillectomy residual OSA is possibly underestimated. In general, by performing polysomnography, a reduction of $51 \%$ in preoperative $\mathrm{AHI}$ can be expected with the intervention of adenotonsillectomy. These are useful data to advise and manage the expectations of parents. It is also a warning to physicians about the importance of following these patients closely after surgery and, although improvements can be seen, patients with Down syndrome will often have a persistent disorder that may require more surgeries or interventions with positive pressure devices CPAP/BiPAP.

Respiratory anomalies are prevalent in patients with trisomy 21 . In one study, $13.8 \%$ of children with trisomy 21 showed airway abnormalities, the most common diagnoses being tracheobronchomalacia and subglottic stenosis. ${ }^{30}$ According to the literature, children with trisomy 21 are at least twice as likely to have laryngomalacia compared with children without the syndrome. Furthermore, almost $50 \%$ may show an epiglottic collapse during a drug-induced sleep endoscopy. However, in the present series, less than $5 \%$ of the patients presented a laryngeal compromise. The reported rate of laryngeal involvement could be due to the lack of a systematic search for laryngeal manifiestations in apparently asymptomatic patients. Concerning treatment, four patients in this series had laryngomalacia, of which three were managed satisfactorily with medical treatment and follow-up. Only one patient required supraglottoplasty, which was satisfactory as well.

The present study makes it possible to demonstrate the variety of otorhinolaryngological pathologies of pediatric patients with trisomy 21 , their morbidity, and the repercussion on the quality of life. All pediatric patients with Down syndrome should be referred systematically for evaluation by an ENT specialist due to the high incidence of manifestations associated with the syndrome in the ears, nose, and throat. Appropriate treatments should be offered to improve the patients' quality of life and offer them greater social inclusion through the improvement of communication skills, the control of infectious diseases, and a reduction in length of hospital stay.

\section{Ethical disclosures}

Protection of human and animal subjects. वेhe authors declare that no experiments were performed on humans or animals for this study.

Confidentiality of data. The authors declare that they have followed the protocols of their work center on the publication of patient data.

Right to privacy and informed consent. The authors have obtained the written informed consent of the patients or subjects mentioned in the article. The corresponding author is in possession of this document.

\section{Conflicts of interest}

The authors declare no conflicts of interest.

\section{References}

1. Asim A, Kumar A, Muthuswamy S, Jain S, Agarwal S. Down syndrome: an insight of the disease. J Biomed Sci. 2015;22:41.

2. Kanamori G, Witter M, Brown J, Williams-Smith L. Otolaryngologic manifestations of Down syndrome. Otolaryngol Clin North Am. 2000;33: 1285-92.

3. Carothers $A D$, Hecht $C A$, Hook EB. International variation in reported live birth prevalence rates of Down syndrome, adjusted for maternabage. J Med Genet. 1999;36:386-93. 
4. Mégarbané A, Ravel A, Mircher $C$, Sturtz F, Grattau Y, Rethoré MO, et al. The $50^{\text {th }}$ anniversary of the discovery of trisomy 21 : the past, present and future of research and treatment of Down syndrome. Genet Med. 2009;11:611-6.

5. Glasson EJ, Sullivan SG, Hussain R, Petterson BA, Montgomery PD, Bittles $\mathrm{AH}$, et al. The changing survival profile of people with Down syndrome: implications for genetic counselling. Clin Genet. 2002;62:390-3.

6. Chin CJ, Khami MM, Husein M. A general review of the otolaryngologic manifestations of Down syndrome. Int $\mathrm{J}$ Pediatr Otorhinolaryngol. 2014;78:899-904.

7. Kong AM, Hurley D, Evans KA, Brixner D, Csoboth C, Visootsak J. A retrospective, longitudinal, claims-based comparison of concomitant diagnoses between individuals with and without Down syndrome. J Manag Care Spec Pharm. 2017;23:761-70.

8. Lau WL, Ko CH, Cheng WW. Prevalence and parental awareness of hearing loss in children with Down syndrome. Chin Med J (Engl). 2015;128:1091-5

9. Kreicher KL, Weir FW, Nguyen SA, Meyer TA. Characteristics and progression of hearing loss in children with Down syndrome. J Pediatr. 2018;193:27-33.

10. Bull MJ; Committee on Genetics. Health supervision for children with Down syndrome. Pediatrics. 2011;128:393-406.

11. Mc Dermott AL, Williams J, Kuo MJ, Reid AP, Proops DW. The role of bone-anchored hearing aids in children with Down syndrome. Int $\mathrm{J} \mathrm{Pe}$ diatr Otorhinolaryngol. 2008;72:751-7.

12. Shott SR, Joseph A, Heithaus D. Hearing loss in children with Down syndrome. Int J Pediatr Otorhinolaryngol. 2001;61:199-205.

13. Ramia M, Musharrafieh U, Khaddage W, Sabri A. Revisiting Down syndrome from the ENT perspective: review of literature and recommendations. Eur Arch Otorhinolaryngol. 2014;271:863-9.

14. Shott SR. Down syndrome: common otolaryngologic manifestations. Am J Med Genet C Semin Med Genet. 2006;142C:131-40.

15. Cole RR, Jahrsdoerfer RA. The risk of cholesteatoma in congenital aural stenosis. Laryngoscope. 1990;100:576-8.

16. Dy AES, Lapeña JFF Jr. External auditory canal dimensions, age, and cerumen retention or impaction in persons with Down syndrome. Ann Otol Rhinol Laryngol. 2018;127:253-7.

17. Bluestone C. Studies in otitis media: Children's Hospital of Pittsburgh University of Pittsburgh Progress Report-2004. Laryngoscope. 2004; 114:1-26.
18. Fortnum $H$, Leighton $P$, Smith $M D$, Brown $L$, Jones $M$, Benton $C$ - et al. Assessment of the feasibility and clinical value of further research to evaluate the management options for children with Down syndrome and otitis media with effusion: a feasibility study. Health Technol Assess. 2014;18:1-147.

19. Rodman R, Pine HS. The otolaryngologist's approach to the patient with Down syndrome. Otolaryngol Clin North Am. 2012;45:599-629.

20. Bacciu A, Pasanisi E, Vincenti V, Giordano D, Caruso A, Lauda L et al. Surgical treatment of middle ear cholesteatoma in children with Down syndrome. Otol Neurotol. 2005;26:1007-10.

21. lino $Y$, Imamura $Y$, Harigai $S$, Tanaka $Y$. Efficacy of tympanostomy tube insertion for otitis media with effusion in children with Down syndrome. Int J Pediatr Otorhinolaryngol. 1999;49:143-9.

22. Crowson MG, Ryan MA, Ramprasad VH, Choi KJ, Raynor E. Intranasal fluticasone associated with delayed tympanostomy tube placement in children with eustachian tube dysfunction. Int J Pediatr Otorhinolaryngol. 2017;94:121-6.

23. Ghadersohi S, Ida JB, Bhushan B, Billings KR. Outcomes of tympanoplasty in children with down syndrome. Int $\mathrm{J}$ Pediatr Otorhinolaryingol. 2017;103:36-40.

24. MRC Multicentre Otitis Media Study Group. Adjuvant adenoidectomy in persistent bilateral otitis media with effusion: hearing and revision surgery outcomes through 2 years in the TARGET randomised trial. Clin Otolaryngol. 2012;37:107-16.

25. Maris M, Verhulst S, Wojciechowski M, Van de Heyning P, Boudewłys $A$ Prevalence of obstructive sleep apnea in children with Down syndrome. Sleep. 2016;39:699-704.

26. Lee CF, Lee CH, Hsueh WY, Lin MT, Kang KT. Prevalence of obstructive sleep apnea in children with Down syndrome: A meta-analysis. I Clin Sleep Med. 2018;14:867-75.

27. Lal C, White DR, Joseph JE, van Bakergem K, LaRosa A. Sleep-disordered breathing in Down syndrome. Chest. 2015;147:570-9.

28. Heubi $\mathrm{CH}$, Meinzen-Derr J, Shott SR, Smith DF, Ishman ASL. Polysomnography in pediatric otolaryngology: if not obstructive sleep apnea, what is it? Otolaryngol Head Neck Surg. 2017;157:1053-9.

29. Farhood Z, Isley JW, Ong AA, Nguyen SA, Camilon TJ, LaRosa AC et al. Adenotonsillectomy outcomes in patients with Down syndrome-and obstructive sleep apnea. Laryngoscope. 2017;127:1465-70.

30. Bertrand P, Navarro H, Caussade S, Holmgren N, Sánchez I. Airway anomalies in children with Down syndrome: endoscopic findings. Pediatr Pulmonol. 2003;36:137-41. 